\title{
Variability of inspired oxygen concentration with nasal cannulas
}

\author{
E A Bazuaye, T N Stone, P A Corris, G J Gibson
}

\begin{abstract}
Background The likely values of inspired oxygen concentration $\left(\mathrm{FIO}_{2}\right)$ of patients with chronic obstructive pulmonary disease breathing via nasal cannulas have not been assessed previously. Methods Seven patients with chronic obstructive lung disease and seven healthy subjects were studied while breathing oxygen via nasal cannulas or fixed performance (Venturi) or uncontrolled (MC) oxygen masks. Breath to breath values of $\mathrm{FIO}_{2}$ were calculated by extrapolation from expired oxygen and carbon dioxide concentrations on the basis of the oxygencarbon dioxide relationship and on the assumption of a respiratory exchange ratio $(R)$ of $0 \cdot 8$.

Results In both groups of subjects the average values of $\mathrm{FIO}_{2}$ with nasal cannulas at 1 and $21 \mathrm{~min}^{-1}$ were of a similar order to those achieved with $24.5 \%$ and $28 \%$ Venturi masks, but variations within and between subjects in both groups breathing via nasal cannulas were considerable and similar to those found with MC masks. In the seven patients with chronic obstructive lung disease breathing via nasal cannulas at $21 \mathrm{~min}^{-1}$ the average $\mathrm{FIO}_{2}$ varied from $23 \cdot 7 \%$ to $34 \cdot 9 \%$.

Conclusions "Typical" values of $\mathrm{FIO}_{2}$ quoted with nasal cannulas can mislead. The results confirm that this mode of oxygen delivery is unsatisfactory if precise control of inspired oxygen is desired.
\end{abstract}

Department of Respiratory Medicine, Freeman Hospital, Newcastle upon Tyne NE7 7DN

E A Bazuaye

T N Stone

P A Corris

G J Gibson

Correspondence to

Dr G J Gibson

(reprints will not be

available)

Accepted 1 March 1992
Nasal cannulas ("prongs") offer a convenient and widely used method of administering oxygen. Although it is acknowledged that the resulting inspired oxygen concentration $\left(\mathrm{FIO}_{2}\right)$ is likely to vary, "typical" values are often quoted. ${ }^{12}$ These are based on a limited number of studies of normal subjects. No systematic attempt has been made to estimate $\mathrm{FIO}_{2}$ and its likely variability in patients with chronic airway disease breathing oxygen via nasal cannulas. Direct measurement of mean $\mathrm{FIO}_{2}$ with

Table 1 Mean (SD) range age and respiratory function in normal subjects and patients

\begin{tabular}{lcc}
\hline & Normal subjects & Patients \\
\hline Age $(\mathrm{yr})$ & $29 \cdot 0(14 \cdot 3)[19-60]$ & $61 \cdot 3(4 \cdot 4)[58-72]$ \\
FÉ $V_{1}$ & & \\
$\quad(1)$ & $4 \cdot 4(0 \cdot 9)[3 \cdot 2-5 \cdot 6]$ & $0 \cdot 7(0 \cdot 3)[0 \cdot 5-1 \cdot 25]$ \\
$\quad \%$ predicted & $112(12 \cdot 4)[95 \cdot 4-134]$ & $29 \cdot 4(5 \cdot 8)[19 \cdot 4-38 \cdot 0]$ \\
Vital capacity $(1)$ & $5 \cdot 2(1 \cdot 3)[3 \cdot 75-7 \cdot 45]$ & $1 \cdot 68(1 \cdot 0)[0 \cdot 8-3 \cdot 7]$ \\
Arterial oxygen saturation $(\%)$ & - & $89 \cdot 2(3 \cdot 7)[84-93]$ \\
\hline
\end{tabular}

such devices is difficult because of problems of streaming of inspired oxygen and because the values vary within each breath, depending on the instantaneous inspiratory flow.

The method of estimating average $\mathrm{FIO}_{2}$ by extrapolation for samples of expired air was introduced by Leigh $^{3}$ and is simple, noninvasive, and reproducible. We have adapted this method to determine $\mathrm{FiO}_{2}$ and its variability $(a)$ breath to breath within subjects and $(b)$ between seven normal subjects and seven stable hypoxaemic patients with chronic obstructive lung disease. Results obtained while they were breathing oxygen via nasal cannulas were compared with those obtained when they were breathing oxygen with Venturi masks and uncontrolled oxygen (MC) masks.

\section{Methods}

THEORETICAL BASIS

The method requires extrapolation on the oxygen-carbon dioxide diagram of Rahn and Fenn ${ }^{4}$ and depends on the principle that samples of expired air contain varying proportions of alveolar and dead space gas; the concentrations of oxygen and carbon dioxide $\left(\mathrm{FO}_{2}, \mathrm{FCO}_{2}\right)$ in the expirate vary inversely and are related by a straight line with a slope $-R$, where $R$ is the respiratory exchange ratio, usually close to $0 \cdot 8$. The point at which the $\mathrm{R}$ line intercepts the oxygen axis - that is, where the carbon dioxide concentration is zero-represents the average inspired oxygen concentration $\left(\mathrm{FIO}_{2}\right)$ for the breath or breaths sampled-that is,

$$
\mathrm{FIO}_{2}=\mathrm{FO}_{2}+\frac{\mathrm{FCO}_{2}}{\mathrm{R}} .
$$

If $\mathrm{R}$ is assumed to be $0 \cdot 8$,

$$
\mathrm{FIO}_{2}=\mathrm{FO}_{2}+1 \cdot 25 \mathrm{FCO}_{2}
$$

SUBJECTS

Seven healthy adult volunteers (five male) and seven patients with chronic obstructive lung disease (five female) were recruited into the study. Details of age and spirometric values are shown in table 1. Informed consent was obtained and approval was given by the local ethics committee.

\section{STUDY PROTOCOL}

The subject sat comfortably on a chair and was asked to breathe as normally as possible with the lips slightly open. Arterial oxygen saturation $\left(\mathrm{SaO}_{2}\right)$ was estimated (in patients only) with an ear oximeter (Biox IIA) after 30 minutes during the breathing of room air and during breathing via the tested devices. Each subject breathed oxygen via each device for at 
Table 2 Variability of inspired oxygen concentration $\left(\mathrm{FIO}_{2}\right)$ in normal subjects and patients

\begin{tabular}{|c|c|c|c|c|c|c|}
\hline & \multicolumn{2}{|c|}{ Venturi masks } & \multicolumn{2}{|c|}{ Nasal cannulas ( $l$ min ') } & \multicolumn{2}{|c|}{$M C$ mask ( $l$ min $\left.{ }^{\prime}\right)$} \\
\hline & $24.5 \%$ & $28 \%$ & 1 & 2 & 2 & 4 \\
\hline \multicolumn{7}{|l|}{ NORMAL SUBJECTS } \\
\hline Average $\mathrm{FIO}_{2}(\%)$ & $25 \cdot 2$ & $30 \cdot 1$ & $26 \cdot 7$ & $30 \cdot 1$ & $37 \cdot 9$ & $46 \cdot 5$ \\
\hline Mean CV within subjects (\%) & 0.9 & 0.6 & 1.9 & $3 \cdot 1$ & $2 \cdot 0$ & $3 \cdot 0$ \\
\hline CV between subjects (\%) & $1 \cdot 2$ & $1 \cdot 7$ & $10 \cdot 1$ & $8 \cdot 6$ & $5 \cdot 8$ & $10 \cdot 5$ \\
\hline \multicolumn{7}{|l|}{ PATIENTS } \\
\hline Average $\mathrm{FIO}_{2}(\%)$ & $25 \cdot 5$ & $30 \cdot 3$ & 28.9 & $29 \cdot 3$ & $\begin{array}{r}38 \cdot 5 \\
28\end{array}$ & $\begin{array}{r}47 \cdot 2 \\
4.0\end{array}$ \\
\hline Mean CV within subjects $(\%)$ & 0.9 & 0.9 & $2 \cdot 9$ & $3 \cdot 1$ & $-3 \cdot 8$ & $\begin{array}{r}4 \cdot 9 \\
13 \cdot 1\end{array}$ \\
\hline CV between subjects (\%) & $2 \cdot 7$ & $3 \cdot 0$ & $14 \cdot 2$ & $13 \cdot 7$ & $11 \cdot 1$ & $13 \cdot 1$ \\
\hline
\end{tabular}

$\mathrm{CV}-$ coefficient of variation.

least five minutes or until the $\mathrm{SaO}_{2}$ was stable, after which at least 10 consecutive expired breaths (average 18 breaths) were sampled. The oxygen and carbon dioxide concentrations were measured continuously by a mass spectrometer (MGA 2000) via a catheter held at the lips in the stream of the exhaled breath. Each subject breathed $(a)$ room air and oxygen via $24.5 \%$ and $28 \%$ Venturi masks (Ventimask MK3, Vickers Ltd) at 2 and $41 \mathrm{~min}^{-1}$ respectively; (b) oxygen at 1 and $21 \mathrm{~min}^{-1}$ via nasal cannulas (Salter Laboratories, Arvin, California-No 1600); and (c) oxygen at 2 and $41 \mathrm{~min}^{-1}$ via an MC mask (Bakelite Xyonite Ltd, Barnsley). The sampling catheter was passed through one of the holes in the mask and held in such a way that it did not occlude the ports. Oxygen was supplied from a standard wall outlet and the calibration of the oxygen flow meter (British Oxygen Company, Harlow, Essex) was confirmed by timed water displacement. The oxygen delivery devices were tested in random order by one observer and were discarded after use. Calibrations of the mass spectrometer and ear oximeter were checked before each device was tested. The analogue signals of $\mathrm{Fo}_{2}, \mathrm{FCO}_{2}$, and $\mathrm{SaO}_{2}$ were recorded on a chart recorder. The values of expired oxygen and carbon dioxide used in the calculation of $\mathrm{FIO}_{2}$ were obtained from the first third of the "alveolar plateau" of each recorded consecutive breath.

The mean $\mathrm{FIO}_{2}$ for each subject with each device was calculated as the average of the extrapolated individual (breath to breath) values.

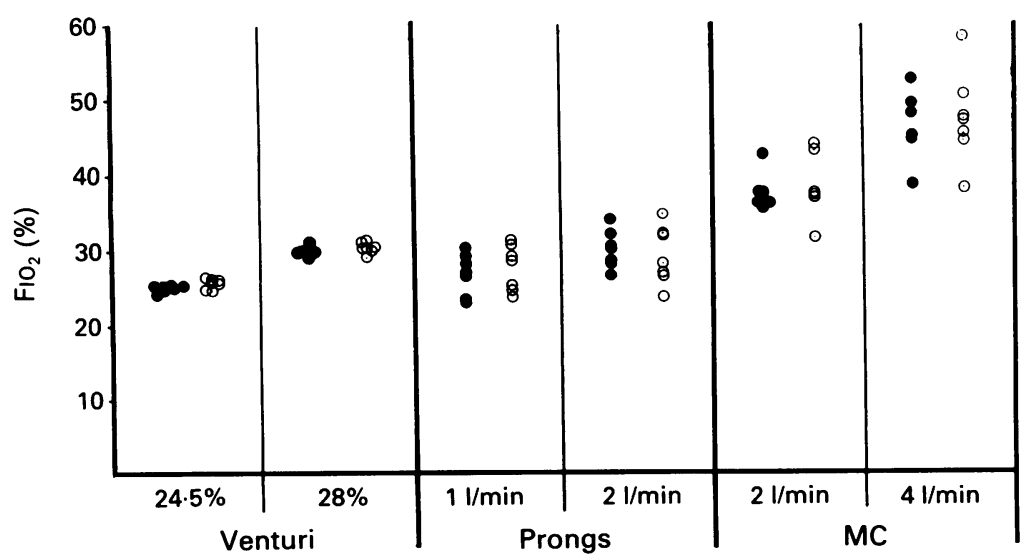

Figure 1 Mean inspired oxygen concentration $\left(\mathrm{F}_{1 \mathrm{O}_{2}}\right.$ ) for individual normal subjects (O) and patients (O) breathing oxygen via various delivery devices. No measurements

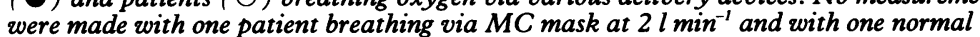
subject breathing via MC mask at 4 l $\mathrm{min}^{-1}$

\section{STATISTICAL ANALYSIS}

The estimated values of $\mathrm{FIO}_{2}$ are shown as means with standard deviations in parentheses; variability in the performance of each device is expressed in terms of the coefficient of variation $(\mathrm{CV})$ both $(a)$ within subjects (that is, variation in extrapolated $\mathrm{FIO}_{2}$ with each breath in a subject) and (b) between subjects (that is, variability in the mean of extrapolated $\mathrm{FIO}_{2}$ from one subject to another). Bias in the estimated values of $\mathrm{FIO}_{2}$ was assessed with the Venturi mask by calculating the mean and SD of the differences in the directly measured inspired oxygen concentrations between room air and oxygen breathing (with this type of mask $\mathrm{FIO}_{2}$ can be sampled directly).

\section{Results}

\section{VARIATION $\mathrm{OF} \mathrm{FIO}_{2}$ WITHIN AND BETWEEN}

SUBJECTS

The coefficients of variation $(\mathrm{CV})$ within and between subjects were similar in normal subjects and patients (table 2). Variations in calculated $\mathrm{FIO}_{2}$ were small with the Venturi mask but showed considerable variation with nasal cannulas both within and between subjects. In the seven patients breathing via nasal cannulas at $21 \mathrm{~min}^{-1}$ the estimated mean $\mathrm{FrO}_{2}$ varied from $23 \cdot 7 \%$ to $34.9 \%$. Variations with the MC mask were of a similar order to those with nasal cannulas.

\section{ESTIMATED MEAN $\mathrm{FIO}_{2}$}

The estimated values of mean $\mathrm{FiO}_{2}$ are shown in summary form in table 2 and for individual subjects in figure 1. The average $\mathrm{FIO}_{2}$ for any device was similar in normal subjects and patients. The estimated mean $\mathrm{FIO}_{2}$ during the breathing of oxygen via the Venturi mask ${ }^{5}$ was close to the nominal values indicated by the manufacturers.

\section{COMPARISON OF ESTIMATED AND DIRECTLY} SAMPLED INSPIRED $\mathrm{FIO}_{2}$

When the subjects were breathing through a Venturi mask the estimated values of $\mathrm{FIO}_{2}$ were slightly, but significantly, greater than the directly measured values and showed a similar mean (SD) bias of $0.38 \%(0.34 \%)$ in normal subjects and $0.33 \%(0.30 \%)$ in the patients.

RELATION OF ESTIMATED $\mathrm{FiO}_{2} \mathrm{TO}^{\mathrm{SaO}}{ }_{2}$

Increases in $\mathrm{SaO}_{2}$ with the various devices were consistent within subjects and showed similar curvilinear relationships (fig 2 ). 


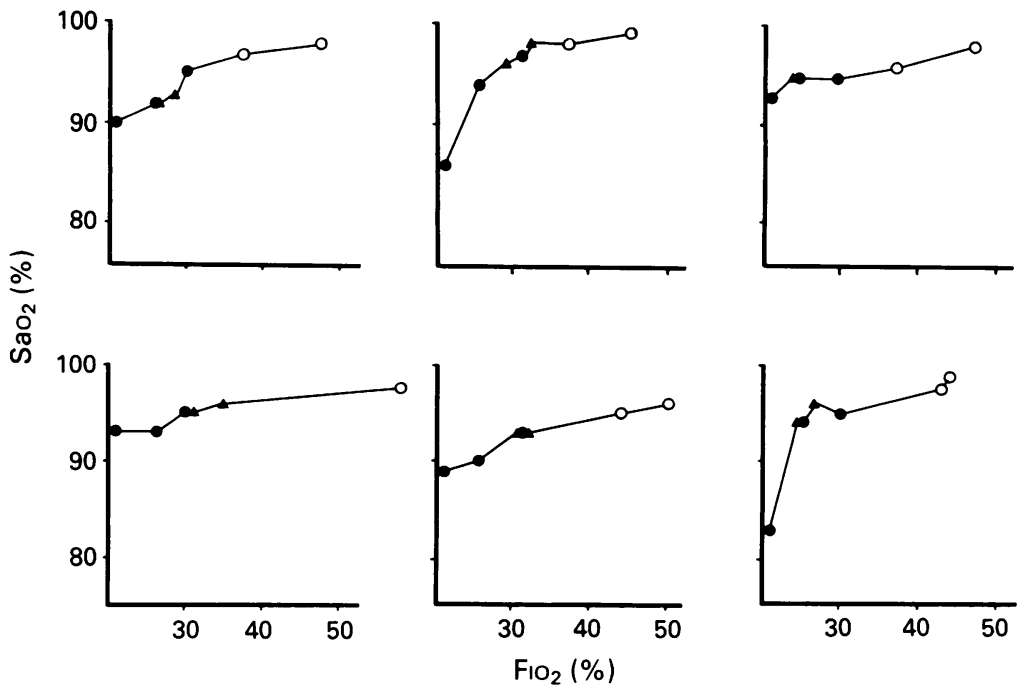

Figure 2 Relations between average estimated inspired oxygen concentration $\left(\mathrm{FIO}_{2}\right)$ and arterial oxygen saturation recorded by ear oximeter in six patients with hypoxaemia breathing air. Values obtained with controlled $\mathrm{FIO}_{2}-$ that is, during the breathing of room air or $24.5 \%$ or $28 \%$ oxygen via Ventimask; $\Delta$ values obtained with nasal prongs; $\bigcirc$ values obtained with MC mask. value of the sampled lung units falls progressively. ${ }^{4}$ For this reason reservations have been expressed over applying this method to patients with lung disease where the pattern of alveolar emptying is abnormal. To minimise this effect we sampled expired gas early in the "alveolar plateau"; the similarity of the estimates of $\mathrm{FIO}_{2}$ obtained in patients and normal subjects supports the validity of this approach, as also do the coherent relationships of arterial oxygen saturation to estimated $\mathrm{FiO}_{2}$ (fig 2). A "typical" $R$ value of 0.8 was assumed but the consistent slight overestimation of $\mathrm{FIO}_{2}$ during breathing via the Venturi mask suggests that this might not have been correct. Calculation shows that the small bias found could be explained if the true $R$ value was on average 0.86 rather than 0.8 .

The study was designed to mimic real life conditions and no attempt was made to control the breathing pattern of individual subjects. Different masks and cannulas were used for each subject, as is normal in clinical practice, and some of the variability between subjects probably reflects minor differences in the performance of individual devices. The variability of $\mathrm{FIO}_{2}$ within and between subjects in clinical practice, however, is likely, if anything, to be underestimated in this study because of the short period over which measurements were made and because the patients were in a stable state. The variability with nasal cannulas during an acute exacerbation is likely to be appreciably greater than that found here.

The results of this study confirm the considerable variability of $\mathrm{FIO}_{2}$ both within and between subjects breathing via nasal cannulas. They emphasise the unpredictable performance of these devices, which are unsatisfactory if precise control of inspired oxygen is desired. $\mathrm{FIO}_{2}$ have serious limitations even in healthy subjects. ${ }^{3}$ Direct sampling of inspired gas close to the mouth will not reflect the average $\mathrm{FIO}_{2}$ with nasal cannulas or MC mask accurately because of local streaming of added oxygen and because of variations in overall ventilation and in the pattern of breathing. Even when inspired gas is sampled in the pharynx or trachea mean values are difficult to obtain because the concentration profile varies with the instantaneous flow. The extrapolation associated with the expired gas sampling method theoretically should give representative mean values for $\mathrm{FIO}_{2}$ in each breath. The method has some limitations, however, and attempts were made to overcome them during this study. If expiration is prolonged, the oxygen and carbon dioxide concentrations in the expired gas approach those of mixed venous blood and the values no longer lie on a unique straight line as the $\mathbf{R}$
1 Bethune DW, Collis JM. An evaluation of oxygen therapy equipment. Thorax 1967;22:221-5.

2 Shapiro BA, Harrison RA, Kaemarck RM, Shapiro BA, Harrison RA, Trout CA, et al. Clinical application of respiratory care. Chicago: Year Book Medical Publishers, 1975:134. devices. Anesthesia 1970;25:210-22.

4 Rahn H, Fenn WO. A graphical analysis of respiratory gas exchange. Washington DC: American Physiological Society, 1955.

5 Bland JM, Altman DC. Statistical methods for assessing agreement between two methods of clinical measurement. Lancet 1986;i:307-10.

6 Campbell EJM. Respiratory failure. The relation between oxygen concentrations of inspired gas and arterial blood. Lancet 1960;ii:10-1.

7 Green IB. Choice of administration of oxygen. BMJ 1967; iii:593-6. tracheal oxygen concentrations with commonly used
3 Leigh JM. Variation in performance of oxygen therapy

8 Gibson RL, Conner PB, Beckham RW, McCraw CP. Actual oxygen equipment. Anaesthesiology 1976;44:71-3. 